\title{
Catheter Ablation of Pediatric Atrioventricular Nodal Re-entrant Tachycardia
}

\author{
SHUNMUGA SUNDARAM PONNUSAMY, MD, DM, PDF (EP), CEPS, ${ }^{1}$ GIRIDHAR MUTHU, MD, DM, ${ }^{1}$ \\ and VIJESH ANAND, MBBS, MD ${ }^{1}$
}

'Department of Cardiology, Velammal Medical College Hospital and Research Institute, Madurai, Tamil Nadu, India

\begin{abstract}
Catheter ablation is considered as the standard treatment for all patients with symptomatic drug-refractory tachyarrhythmia. The safety and efficacy of the procedure in the adult population is well-established. Due to the small size of the patient and difficulty in attaining venous access, infants are rarely subjected to radiofrequency ablation. Here, we report a case of drug-refractory $A V$ nodal re-entrant tachycardia in a two-year-old child. Radiofrequency ablation was performed with a 5-Fr sized medium-curve ablation catheter deployed at the slow pathway region where a fractionated A-wave with slow-pathway potential was recorded. After ablation, no recurrence of SVT at the end of 12 months of follow-up was observed.
\end{abstract}

KEYWORDS. AVNRT, pediatric arrhythmia, radiofrequency ablation.
ISSN 2156-3977 (print) ISSN 2156-3993 (online) CC BY 4.0 license

(c) 2020 Innovations in Cardiac Rhythm Management

\section{Introduction}

Although catheter ablation for cardiac arrhythmia has been performed in adult populations since the early 1980s, ablation for cardiac arrythmias in the pediatric population was not attempted until the early 1990s. ${ }^{1,2}$ Supraventricular tachycardias (SVTs) are the most commonly encountered substrates, among which, those related to the accessory pathway account for $75 \%$ of cases in older children and $95 \%$ of cases among infants. Atrioventricular (AV) node reentrant tachycardia (AVNRT) is a less frequent (12\%) occurrence. ${ }^{3,4}$ Most of these arrhythmias respond to medical management and catheter ablation is reserved for drug-refractory cases only. Cryoablation is the preferred modality for infants and young children as it reduces the risk of AV blockage. Herewith, we report the case of a two-year-old child with drug-refractory AVNRT with repeated hospitalization for the arrhythmia who was subsequently treated by radiofrequency $(\mathrm{RF})$ ablation.

\footnotetext{
The authors report no conflicts of interest for the published content. Address correspondence to: Dr. Shunmuga Sundaram Ponnusamy, MD, DM, PDF (EP), CEPS, Department of Cardiology Velammal Medical College Hospital and Research Institute, Velammal Village, Airport Ring Road, Madurai 625009, Tamil Nadu, India.

Email: shunmuga.pgi@gmail.com.
}

\section{Case report}

A two-year-old child weighing $10 \mathrm{~kg}$ was referred for catheter ablation for drug-refractory SVT. Her mother reported a history of frequent hospitalizations despite maximal doses of oral verapamil and $\beta$-blocker medication. Electrocardiography (ECG) during sinus rhythm did not show pre-excitation. Echocardiography ruled out structural heart disease. A narrow QRS tachycardia at the rate of $260 \mathrm{bpm}$ was documented during an SVT episode (Figure 1). In view of recurrent hospitalization despite maximal medical management, an option of catheter ablation was discussed. Informed consent was obtained in view of the nonavailability of cryoablation and the risk of AV blockage.

RF ablation was performed under intravenous sedation. Twelve-lead ECG and intracardiac electrograms were continuously recorded using an electrophysiology system (Abbott Laboratories, Chicago, IL, USA). Venous access was obtained from both the right femoral vein and the left femoral vein. Baseline sinus rhythm showed normal AH (94 ms) and HV (39 ms) intervals (Figure 2A). Tachycardia could be easily induced by programmed atrial stimulation at $400 \mathrm{~ms}$ (S1), $350 \mathrm{~ms}$ (S2), and $230 \mathrm{~ms}$ (S3) with an AH jump. The tachycardia cycle length was $230 \mathrm{~ms}$ and coronary sinus electrograms showed a concentric atrial 


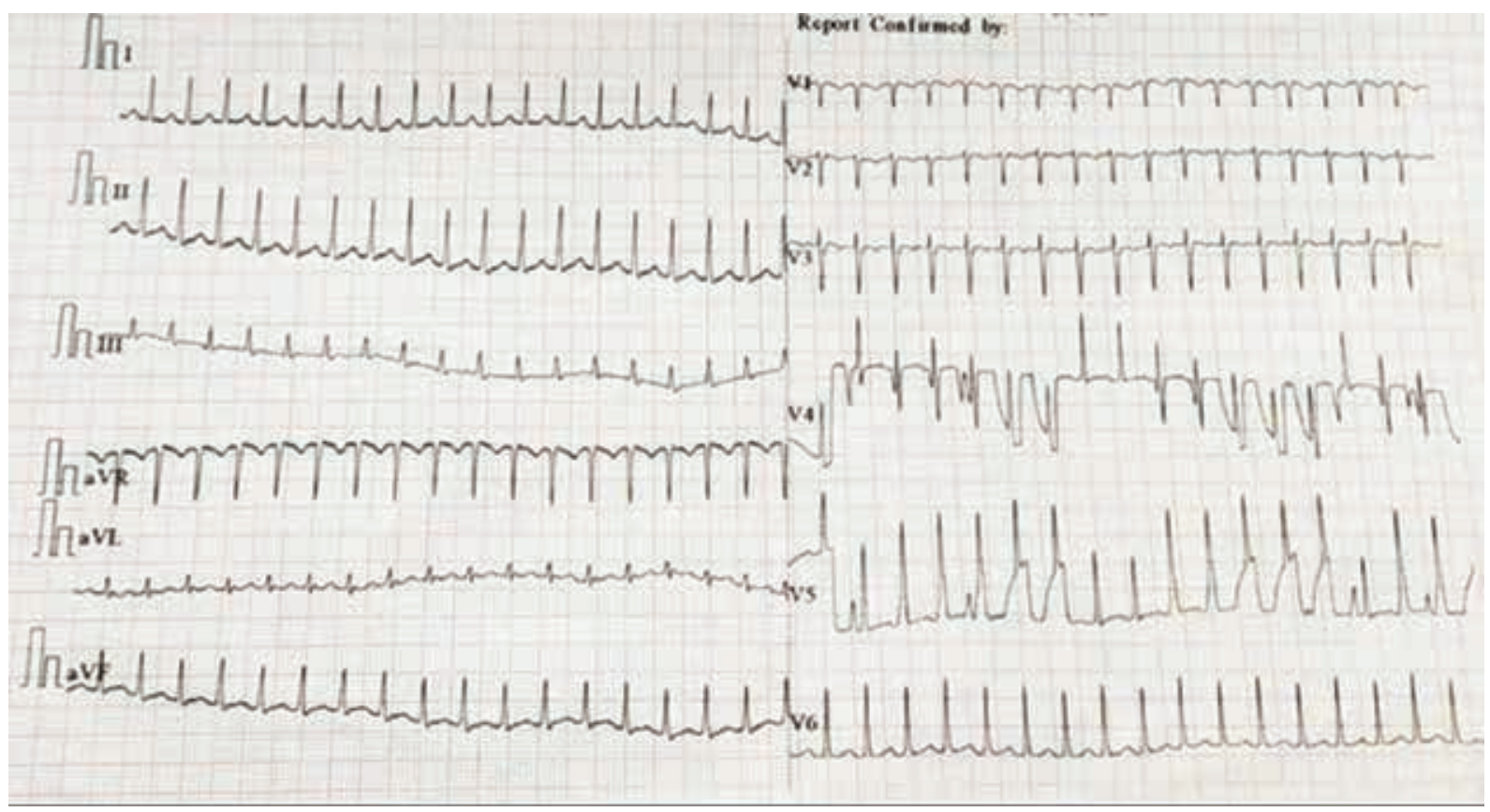

Figure 1: ECG taken during tachycardia showed a regular narrow QRS tachycardia with a pseudo-S-wave in lead II and pseudo-R-wave in lead aVR suggestive of AVNRT.

A

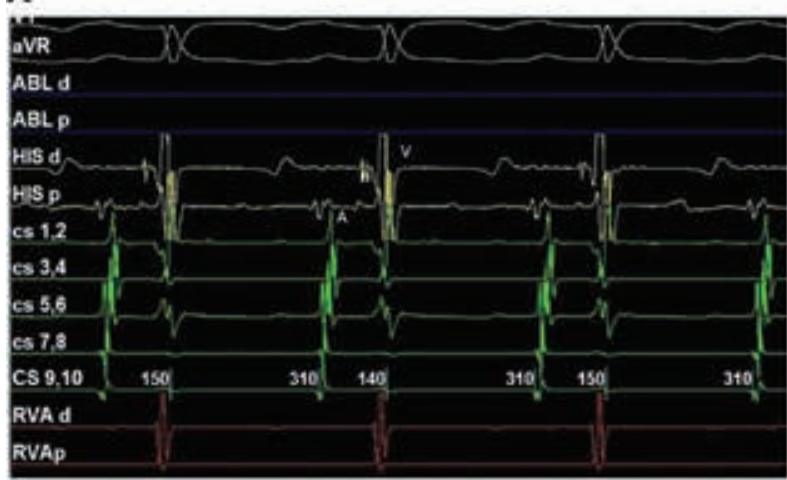

c

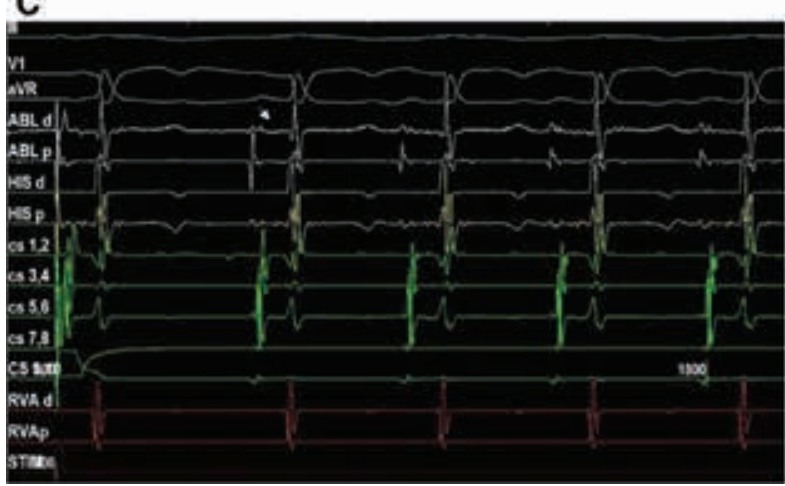

B

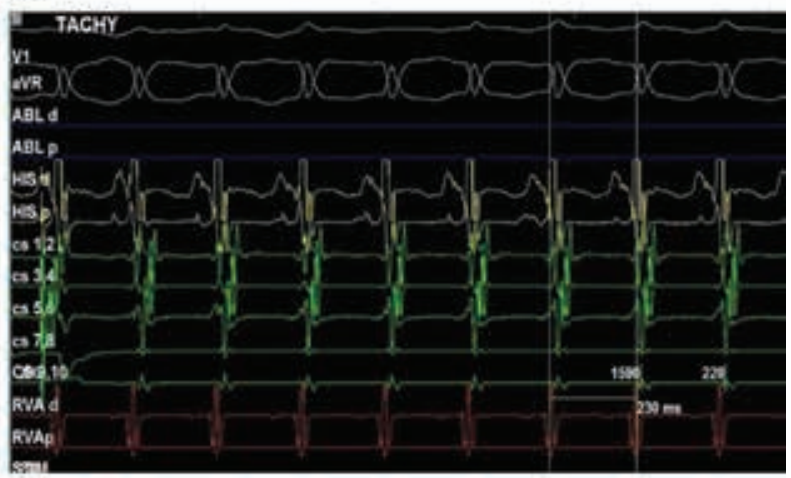

D

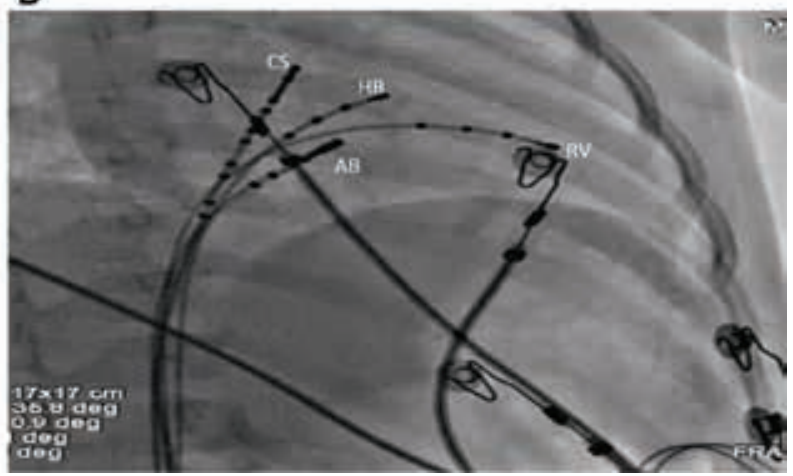

Figure 2: A: Baseline $\mathrm{AH}$ and HV intervals were $94 \mathrm{~ms}$ and $39 \mathrm{~ms}$. B: AVNRT with a cycle length of $230 \mathrm{~ms}$ with a concentric activation pattern in the coronary sinus. C: Ablation catheter showing a fractionated atrial signal with slow-pathway potential (white arrowhead). D: Ablation catheter at the anterior margin of the coronary sinus in the right anterior oblique fluoroscopy view. CS: coronary sinus; $\mathrm{HB}$ : His bundle; $\mathrm{AB}$ : ablation catheter; RV: right ventricle. 

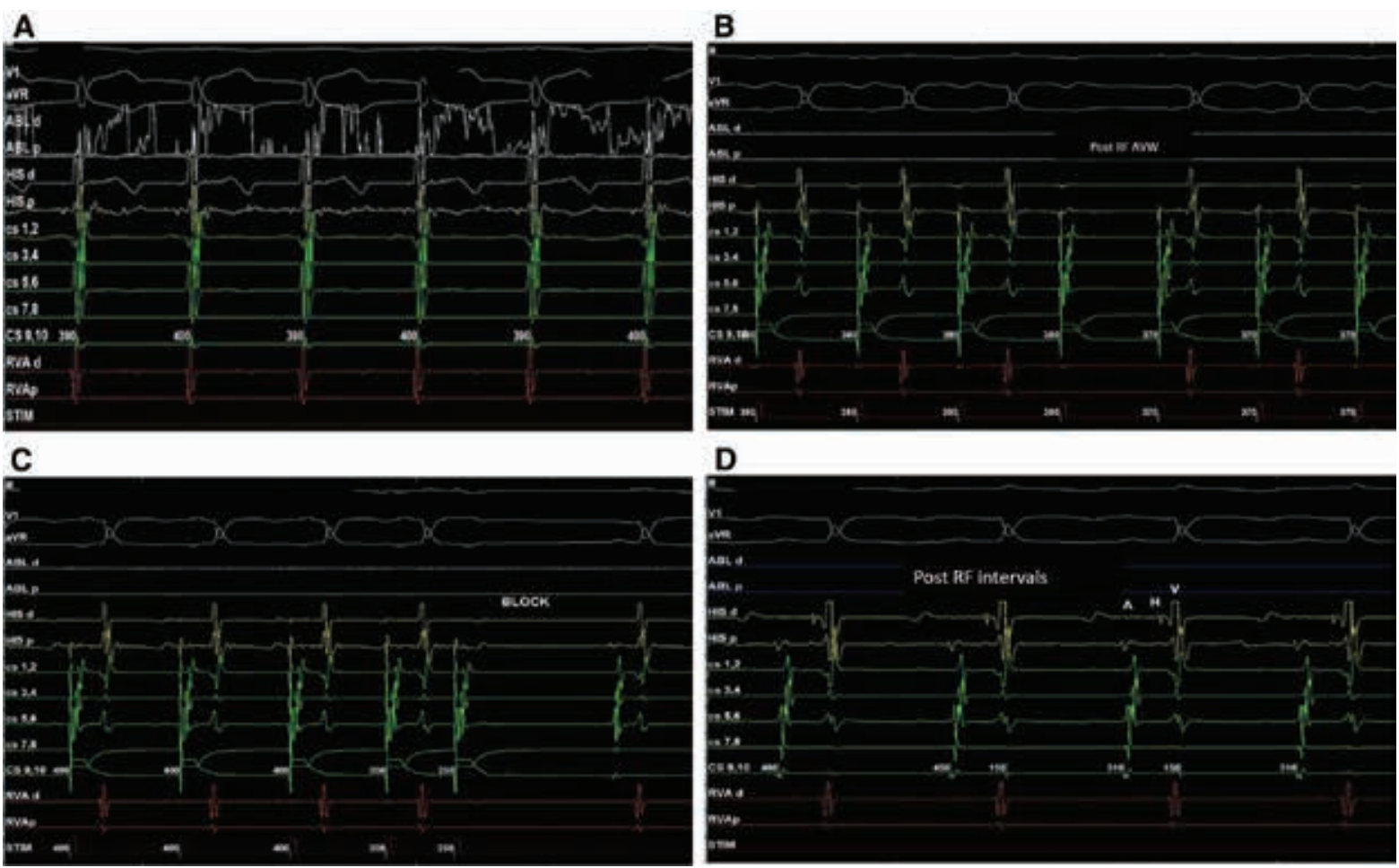

Figure 3: A: Junctional rhythm during RF ablation at a 400-ms cycle length. B: Post-RF ablation incremental atrial pacing showed Wenckebaching at a 380-ms cycle length. C: Programmed atrial stimulation showed AH block at 400 ms (S1), 350 ms (S2), and $250 \mathrm{~ms}$ (S3). D: Postablation AV-nodal conduction remained intact with AH and HV intervals of 94 ms and 40 ms.

activation pattern (Figure 2B). The ventricular entrainment protocol confirmed AVNRT.

Ablation at the posterior part of Koch's triangle with a fractionated atrial signal with slow-pathway potential (Figures 2C and 2D) using a 5-French (Fr) medium-curve RF ablation catheter (Abbott Laboratories, Chicago, IL, USA) resulted in good junctional rhythm at the 400-ms cycle length ( $30 \mathrm{~W}$ power gradually increased to $50 \mathrm{~W}$, temperature of $50^{\circ} \mathrm{C}$ for 60 seconds) (Figure 3A). The total fluoroscopy time was five minutes and the procedure lasted for 30 minutes. Postablation tachycardia could not be induced despite an aggressive induction protocol. Incremental atrial pacing showed AV Wenckebaching at a 380-ms cycle length, confirming the elimination of slow-pathway conduction (Figure 3B). Fast-pathway conduction remained intact as programmed atrial stimulation revealed $\mathrm{AH}$ block at $400 \mathrm{~ms}$ (S1), $350 \mathrm{~ms}$ (S2), and $250 \mathrm{~ms}$ (S3) (Figure 3C). The AH and HV intervals after ablation were $94 \mathrm{~ms}$ and $40 \mathrm{~ms}$, respectively (Figure 3D). The child was discharged the next day without any complications and remained symptom-free at 12 months after the procedure.

\section{Discussion}

Drug-refractory SVTs are rare during childhood. The most common arrhythmias are accessory pathway-mediated AVNRT. Although there are several reports of successful ablation of orthodromic AV-reciprocating tachycardia in infants, AVNRT is rare. Our case is unique in that the AVNRT was drug-refractory despite optimal therapy and RF ablation was performed instead of cryoablation. Two major challenges exist with this protocol: (1) obtaining venous access and (2) avoiding causing injury to the AV node while ablating the slow pathway. In this present case, we restricted our venous access to the femoral vein (two punctures on each side, using a 4-Fr quadripolar catheter and a 5-Fr ablation catheter). Venous Doppler imaging was performed before discharge and ruled out venous thrombosis. To avoid AV nodal block during ablation, it was deemed safe to target fractionated atrial signals with an $\mathrm{AV}$ ratio of 1:10 (Figure 2C) and to stop ablating when there existed a fast junctional rhythm at a cycle length of less than $300 \mathrm{~ms}$.

AVNRT is the second most-encountered SVT in the pediatric population. The AV node consists of two pathways: the fast pathway, which is composed of transitional cells in the region extending from the compact node to the anterior aspect of Koch's triangle, and a slow pathway composed of a deeper inferoposterior extension..$^{5}$ AVNRT can be safely cured by slow-pathway modification in the posterior aspect of Koch's triangle. Cryoablation is the modality of choice in infants and children as it can reduce the incidence of AV block. However, we had to employ RF energy as cryoablation is not yet available in our region.

The most common challenge that we come across in the pediatric population is the small size of the heart and the 
varied anatomy associated with congenital heart diseases. ${ }^{6}$ The varied intracardiac and venous anatomy often makes it difficult to ablate in this population. In many centers, the procedures are modified with the usage of fewer catheters than in the adult population and the adoption of transesophageal catheters to pace the left atrium and 4-Fr ablation catheters to avoid vascular complications. The reported complications during pediatric RF ablation include complete heart block and thrombus formation. Cardiac perforation and death are rare complications and are more frequent in correlation with smaller patient sizes and left-sided procedures. Blaufox et al. ${ }^{7}$ showed the feasibility of catheter ablation for cardiac arrhythmias in small children weighing less than $15 \mathrm{~kg}$. Of $268 \mathrm{RF}$ catheter ablation procedures, 18 were performed in 14 patients weighing less than $15 \mathrm{~kg}$. Orthodromic AVRT was noted in nine patients, atrial tachycardia was seen in one patient, and ventricular tachycardia was found in four patients. The complications reported included pericardial effusion, mitral regurgitation, and myocardial infarction. In the AVRT subgroup, the indexed total application time trended higher during complicated as compared with during uncomplicated procedures (40.6 versus $6.6 \mathrm{~s} / \mathrm{kg}$ ). The authors concluded that RF ablation can be successful in small children; however, complications appear to be related to the RF dose indexed for body size. Elsewhere, the MAP-IT registry ${ }^{8}$ highlighted the safety of pediatric ablation with a significant reduction in radiation exposure among the 1,417 procedures done. Cryoablation was performed in one-quarter of the cases. Two percent of the patients were younger than three years of age and their success rate and complications were not different from those of older patients.

Despite all these challenges and complications, however, catheter ablation for AVNRT is a safe procedure in the pediatric population when performed by experienced hands.

\section{Conclusion}

AVNRT is the second most common variety of SVT in children. Drug therapy remains the first option for treating pediatric arrhythmias. In rare instances where drugs are not effective in controlling the arrhythmia, RF catheter ablation can be safely performed while safeguarding the AV node. Although single case results cannot be extrapolated to the general population, further studies could provide additional insights and confirm the safety of RF ablation in toddlers.

\section{References}

1. Van Hare GF, Velvis H, Langberg JJ. Successful transcatheter ablation of congenital junction ectopic tachycardia in a tenmonth-old infant using radiofrequency energy. Pacing Clin Electrophysiol. 1990;13(6):730-735.

2. Tanel RE, Walsh EP, Triedman JK, Epstein MR, Bergau DM, Saul JP. Five-year experience with radiofrequency catheter ablation: implications for management of arrhythmias in pediatric and young adult patients. J Pediatr. 1997;131(6):878-887.

3. Kugler JD, Danford DA, Houston KA, Felix G; Pediatric Radiofrequency Ablation Registry of the Pediatric Radiofrequency Ablation Registry of the Pediatric Electrophysiology Society. Pediatric radiofrequency catheter ablation registry success, fluoroscopy time, and complication rate for supraventricular tachycardia: comparison of early and recent eras. J Cardiovasc Electrophysiol. 2002;13(4): 336-341.

4. Van Hare GF, Javitz H, Carmelli D, et al. Prospective assessment after pediatric cardiac ablation: recurrence at 1 year after initially successful ablation of supraventricular tachycardia. Heart Rhythm. 2004;1(2):188-196.

5. Cohen MI, Wieand TS, Rhodes LA, Vetter VL. Electrophysiologic properties of the atrioventricular node in pediatric patients. J Am Coll Cardiol. 1997;29(2):403-407.

6. Silka MJ, Halperin BD, Hardy BG, McAnulty JH, Kron J. Safety and efficacy of radiofrequency modification of slow pathway conduction in children less than 10 years of age with atrioventricular node reentrant tachycardia. Am J Cardiol. 1997;80(10):1364-1367.

7. Blaufox AD, Paul T, Saul JP. Radiofrequency catheter ablation in small children: relationship of complications to application dose. Pacing Clin Electrophysiol. 2004;27(2):224-229.

8. Dubin AM, Jorgensen NW, Radbill AE, et al. What have we learned in the last 20 years? A comparison of modern era paediatric and congenital catheter ablation registry to prior paediatric ablation registries. Heart Rhythm. 2019;16(1): 57-63. 